\title{
MANCHESTER
}

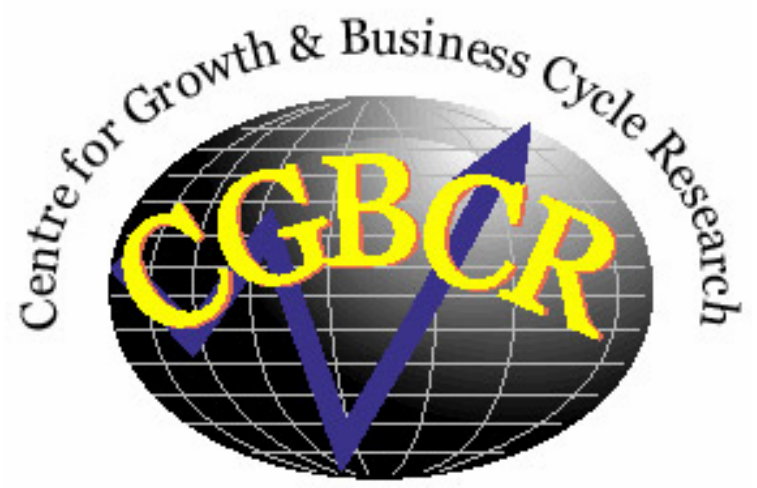

Discussion Paper Series

\section{Monetary policy with heterogeneous and misspecified expectations \\ By}

\section{Michele Berardi}

Centre for Growth and Business Cycle Research, Economic Studies,

University of Manchester, Manchester, M13 9PL, UK

November 2006

Number 081

Download paper from:

http://www.ses.man.ac.uk/cgbcr/discussi.htm 


\title{
Monetary policy with heterogeneous and misspecified expectations
}

\author{
Michele Berardi* \\ School of Social Sciences \\ University of Manchester
}

November 2006

\begin{abstract}
In recent literature on monetary policy and learning, it has been suggested that private sector's expectations should play a role in the policy rule implemented by the central bank, as they could improve the ability of the policymaker to stabilize the economy. Private sector's expectations, in these studies, are often taken to be homogeneous and rational, at least in the limit of a learning process. In this paper, instead, we consider the case in which private agents are heterogeneous in their expectations formation mechanisms and hold heterogeneous expectations in equilibrium. We investigates the impact of this heterogeneity in expectations on central bank's policy implementation and on the ensuing economic outcomes.

Key words: Adaptive learning, expectations formation, heterogenous expectations, misspecifications, monetary policy.

JEL classification: E52, C62, D83.
\end{abstract}

\section{Introduction}

Recent works in monetary policy and learning have suggested that private sector's expectations should be taken into account in the policy rule implemented by the

${ }^{*}$ Contact information: School of Social Sciences, University of Manchester, Dover Street Building (New Wing), Manchester M13 9PL (UK). Email: Michele.Berardi@manchester.ac.uk 
central bank (CB), as this practice could improve the ability of the policymaker to stabilize the economy, especially when agents are learning. In particular, it has been shown that an expectations based policy in a New Keynesian framework is able to ensure that the economy has a unique stable equilibrium and that the equilibrium is learnable by agents (Evans and Honkapohja, 2003a and 2003b).

Unfortunately, no such a variable as "private sector's expectations" exists in reality, as expectations for future economic conditions can vary quite significantly across economic agents. The aim of this paper is to start studying the implications of heterogeneity of expectations and learning dynamics for monetary policy. We do it in a very simple setting, and we assume only two groups of agents that simultaneously learn using different perceived laws of motion (PLMs): one group is endowed with the correct model for the economy, that in the limit of the learning process possibly leads to correct (rational) expectations; and the other is endowed with a misspecified model, one that neglects one or more variables and that even in a learning equilibrium does not allow for rational expectations. As explained later, we can think of these two groups of agents as representing informed and uniformed agents, the former in particular aware of the existence of a central bank and of its role in the economy, while the latter neglectful of it.

Literature on learning in monetary economics often assumes that agents are homogeneous and endowed with a correctly specified model, i.e., one that includes all and only those variables that actually play a role in determining the dynamics of the economy. This assumption, though representing a useful benchmark, does not take into account the fact that private agents, when implementing their learning activity, are like econometricians that need to decide which model to use. Guided by their idiosyncratic beliefs, they are likely to start by selecting a few key variables that they think are necessary to represent the dynamics of the economy and will then change their choice only if this is rejected by data. ${ }^{12}$ If all agents were initially to make use of an overparameterized model, one that includes more variables than those actually involved in the data generating process, they could learn over time to discard the unnecessary variables and finally end up with the correct model; but if instead they were to start out by using an underparameterized model, one that neglects one or more variables actually necessary for the

\footnotetext{
${ }^{1}$ Of course, agents would also need to choose the functional form to relate those variables together. For simplicity, we consider only a linear model.

${ }^{2}$ Here we abstract from considerations of interaction in expectation formation. Each agent forms expectations independently from others, a part for the indirect interactions that happen through economic outcomes.
} 
description of the economy, there is the possibility that they will never realize to be using a misspecified model, as long as an orthogonality condition between the forecasting errors and the variables included in the agents' information set holds. The economy would end up in what is called a restricted perceptions equilibrium (RPE), where agents do not realize to be using a misspecified model. An application of this concept to a New Keynesian monetary model has been studied by Berardi and Duffy (2007), where the misspecification in the agents' model is linked to the degree of transparency of the CB.

In this paper we introduce the possibility of different agents choosing different models, and in particular we consider the case in which the population is split in two groups: type-1 agents, who use a correct model of the economy in their learning process; and type-2 agents, who instead are constrained to use an underparameterized model (in particular, one that neglects lagged values of the endogenous variables). It follows that, given their PLM, type-2 agents can not possibly learn the true data generating process for the system, and the economy can not converge towards a rational expectations equilibrium (REE). Nevertheless, we show that the economy can still converge to an equilibrium in which beliefs of all agents are confirmed by data, in the weaker sense of forecasting errors being uncorrelated with regressors in the PLM. This is an important result, because if the economy were not to converge, agents would probably dismiss the models they were using and try different ones. Instead, if the economy converges towards an equilibrium, it is likely that agents will not consider to use different models, and therefore the economy can get stuck in an equilibrium different form the REE.

While the two types of expectations that coexist in equilibrium differ in their degree of accuracy, they both satisfy a consistency condition that makes them an equilibrium point for the relative learning scheme. We call this equilibrium an Heterogeneous Expectations Equilibrium (HEE), where one group of agents holds rational expectations while the other has expectations that are rational only in a limited sense, in that they are the best forecasts agents can make given the misspecified model they are using. We provide conditions for the existence and learnability (E-stability) ${ }^{3}$ of such an equilibrium in a basic New Keynesian monetary model under different policy specifications, and then compare the properties of different equilibria in terms of output and inflation volatility. In particular, we want to investigate what happens if the CB doesn't realize that there exists heterogeneity in private sector's expectations and uses only one type of expectations

\footnotetext{
${ }^{3}$ For a detailed presentation of the E-stability concept and its relation with real time learning, see Evans and Honkapohja (2001).
} 
when deciding its policy rule.

The structure of the paper is as follows: Section 2 presents the literature this paper builds on; Section 3 develops the model, finds the HEE under different policy specifications and compares them; Section 4 concludes.

\section{Some background theory}

\subsection{Expectations and monetary policy}

When the CB sets its policy instrument, the short term interest rate, it usually takes into account various indicators. Among them, it has been suggested, there should be private expectations about future output and inflation, especially when agents are not fully rational. By responding to private sector's expectations, in fact, the $\mathrm{CB}$ can induce an otherwise unstable REE to be E-stable, i.e. learnable (see Evans and Honkapohja, 2003a and 2003b).

This argument relies on the existence of common expectations among private agents and on the ability of the $\mathrm{CB}$ to observe the value of those expectations. In reality, though, we observe a wide spectrum of expectations/forecasts across the many agents that form the private sector, and reducing this wide heterogeneity to its mean value, to be taken as a sort of representative agent's expectations, might lead us to neglect some important features of the dynamics of the economy.

Another issue that has to be considered is the degree of awareness of the $\mathrm{CB}$ to this heterogeneity in expectations: the $\mathrm{CB}$ might be able to observe the whole range of expectations in the economy, and therefore be able to take this heterogeneity fully into account; or instead it might be able to observe only a subset of them, and mistakenly believe that those expectations well represent the whole population. It is thus important to understand whether the policymaker, by responding to different expectations, can generate different outcomes for the economy. If the answer is yes, heterogeneity becomes relevant for policymaking, and must be taken seriously into account.

In addition, the policy rule implemented by the $\mathrm{CB}$ will impact not only on macroeconomic aggregates but also on agents' expectations formation processes, thus causing a feedback loop that can have rich consequences for the dynamics of the economy.

We address these issues in a simple setting, a standard New Keynesian model populated by two groups of agents independently forming their expectations. Each group is endowed with a model and in every period it updates the parameter es- 
timates through recursive least squares techniques, then using the new values to project the model one-step-ahead in the future and make forecasts about output and inflation. The CB uses an expectations-based policy rule (Evans and Honkapohja, 2003a, 2003b), with coefficients optimally derived from the minimization of its loss function. Given the heterogeneity of expectations, the rule could in principle be implemented using either an average of the two different values or instead only one of the two. This choice will affect the actual law of motion for the economy.

We want to stress here that the policy rule we consider is not fully optimal under learning, in the sense that if the policymaker were to know the expectations formation mechanism of agents, he could use this information to devise a better policy. See Ferrero (2004) and Molnar and Santoro (2005) for a derivation of such a policy with homogeneous agents. But the expectations based policy rule proposed by Evans and Honkapohja, and that we use here, is optimal in a restricted sense, when the CB does not know the specific mechanism used by private agents to from their expectations and takes those expectations as given in the optimization problem. It is important to note that this policy is derived under commitment, and therefore features history dependency, a property that is considered necessary by Woodford (2000) for optimal policy in forward-looking systems, when expectations about the future affect the current state of the economy. Similar history dependent policy rules could be obtained through other procedures, for examples by modifying the central bank loss function.

\subsection{Heterogeneous Expectations Equilibrium}

There are two groups of agents in the economy we consider, learning independently from each other through the recurrent estimation of a model economy. In order to understand what happens as time goes by and the two groups of agents update their beliefs about the parameters in their models, we need to find simultaneously the limiting behavior of the two learning schemes, as they indirectly interact through the impact of agents' expectations on macroeconomic outcomes. Given that the two parts are using different models, one of which is underparameterized, beliefs of the two groups will not converge towards each other, and heterogeneity will be retained in the limit. Still, we can look for an equilibrium outcome, one in which all agents have stopped updating their parameters' estimates: this will happen, under RLS learning, when an orthogonality condition between the variables included in the information set of each group and the fore- 
cast errors of that group holds. Agents endowed with the correct model for the economy will hold in equilibrium rational expectations, while agents using the underparameterized model will hold instead misspecified beliefs, which nevertheless are optimal given the model they are using.

The HEE we use is a refinement of the concept introduced by Branch and McGough (2005), and has been derived and analyzed for an univariate model in Berardi (2007). The necessary extensions for the multivariate case are developed here.

The difference with the HEE proposed by Branch and McGough (2005) is that in that case one group of agents is endowed with adaptive static expectations and therefore can not learn from experience: no orthogonality condition between forecast errors and explanatory variables holds for them, and their expectations are persistently falsified by data. We therefore think that this situation can not be regarded as an equilibrium from a learning perspective.

\section{Monetary policy under heterogenous learning and mis- specifications}

\subsection{The model economy}

We will carry out our analysis using a New Keynesian monetary model closed with an expectations based policy rule derived under commitment. This type of rule has been proposed first in Evans and Honkapohja (2003b), where the authors show that the minimum state variable (MSV) REE in this same New Keynesian setting is determinate and E-stable when the CB implements a policy that responds to private expectations. For the learning process to converge to the REE, of course, private agents must use a correctly specified PLM in their learning process. Berardi and Duffy (2007) show that when the PLM employed by agents is underparameterized and neglects the dependency of the endogenous variables on lagged output, the economy can still converge to an RPE that is stable under least squares learning (E-stable).

Here we introduce heterogeneity among private agents, and assume that there are two groups, using different models for their learning processes. One group uses a correctly specified model, while the other uses a misspecified (specifically, underparameterized) PLM: given this constraint, the economy can not converge towards a symmetric equilibrium and the beliefs of the two groups remain different even asymptotically. But we can show that the economy can still converge to 
an equilibrium, an HEE, where all agents stop updating their estimates for the parameters in their model.

Heterogeneity between the learning processes of the two parts arises here because of asymmetric information between agents: while one group knows what kind of policy the $\mathrm{CB}$ is implementing and what are the consequences of that policy in terms of the dynamics of the system, the other group does not have (or disregards) this piece of information and thus neglects those variables (here, lagged output) that affect the current state of the economy only because of CB's policy. The rationality for this is that there might be limits in CB's ability to communicate clearly its policy to the public, so that part of the private sector might remain unaware of some details of the policy. Alternatively, part of the private sector could disregard the CB's claim to be acting under commitment because aware of the time inconsistency problem and of the temptation for a $\mathrm{CB}$ to surprise the public by announcing a commitment policy but then acting under discretion.

The model we use is a standard forward-looking New Keynesian model, as presented, e.g., in Clarida, Gali and Gertler (1999). The two structural equations are:

$$
\begin{aligned}
y_{t} & =-\varphi\left(i_{t}-E_{t}^{A} \pi_{t+1}\right)+E_{t}^{A} y_{t+1}+g_{t} \\
\pi_{t} & =\lambda y_{t}+\beta E_{t}^{A} \pi_{t+1}+u_{t} .
\end{aligned}
$$

Equation (1) is a forward-looking IS equation, obtained by log-linearizing the consumption Euler equation that arises from the household's optimal saving decision. $y_{t}$ is the output gap, the deviation of the output from its potential level, $i_{t}$ is the interest rate, which is here taken to be the policy instrument, and $\pi_{t}$ is the inflation rate.

Equation (2) is a forward-looking Phillips curve derived under the assumption of staggered nominal price setting by optimizing monopolistically competitive firms. Individual pricing decisions are aggregated and the ensuing relation is log-linearized around the steady state.

$E_{t}^{A}$ indicate average expectations, i.e.

$$
E_{t}^{A}=\omega E_{t}^{1}+(1-\omega) E_{t}^{2}
$$

where $\omega$ is the proportion of agents holding type- 1 expectations.

There is no agreement in the literature about the values to assign to the parameters in the structural equations. Prominent examples of values that have 
been used in the literature are: Clarida, Gali and Gertler (2000): $\varphi=1, \lambda=.3$, $\beta=.99 ;$ McCallum and Nelson (1999): $\varphi=.164, \lambda=.3, \beta=.99$; and Woodford (1999): $\varphi=1 / .157, \lambda=.024, \beta=.99$. We will refer to these calibrations respectively as CGG, MN and $\mathrm{W}$.

The two shocks follow $\mathrm{AR}(1)$ processes:

$$
\begin{aligned}
g_{t} & =\mu g_{t-1}+\tilde{g}_{t} \\
u_{t} & =\rho u_{t-1}+\tilde{u}_{t}
\end{aligned}
$$

with damping coefficients $\mu$ and $\rho$ between 0 and $1 . g_{t}$ represents a demand shock coming from potential output or government expenses, while $u_{t}$ represents any cost push shocks to marginal costs not entering the Phillips curve through $y_{t} \cdot \tilde{g}_{t}$ and $\tilde{u}_{t}$ are white noise processes with zero mean and variances $\sigma_{\tilde{g}}^{2}$ and $\sigma_{\tilde{u}}^{2}$ respectively.

We close the model by specifying a policy rule for the interest rate $i_{t}$. We use an expectations based policy rule derived under the assumption of commitment: in particular we take a timeless-perspective approach and use the time invariant policy rule that can be obtained by discarding the optimality condition for the starting period. One important consequence of this policy rule is to make lagged output a relevant variable for the dynamics of current inflation and output. The specific rule we use is

$$
i_{t}=\phi_{1} y_{t-1}+\phi_{2} E_{t}^{*} \pi_{t+1}+\phi_{3} E_{t}^{*} y_{t+1}+\phi_{4} g_{t}+\phi_{5} u_{t} .
$$

where $E_{t}^{*}$ indicates the expectations used by the CB in the policy, which could in principle be either $E_{t}^{A}, E_{t}^{1}$ or $E_{t}^{2}$. The $\phi_{i}$ coefficients are constrained by the optimality conditions for the minimization problem of a loss function that is quadratic in deviations of inflation and output from target values, and are as follows:

$$
\begin{aligned}
& \phi_{1}=\frac{-\alpha}{\varphi\left(\alpha+\lambda^{2}\right)}, \phi_{2}=1+\frac{\lambda \beta}{\varphi\left(\alpha+\lambda^{2}\right)}, \\
& \phi_{3}=\frac{1}{\varphi}, \phi_{4}=\frac{1}{\varphi}, \phi_{5}=\frac{\lambda}{\varphi\left(\alpha+\lambda^{2}\right)},
\end{aligned}
$$

where $\alpha$ is the weight on output in the loss function for the CB. ${ }^{4}$ As pointed out before, this policy is not fully optimal in case the CB knows the expectations formation process of private agents, as in that case the policymaker could take

\footnotetext{
${ }^{4}$ The specific form of the loss function is $\sum_{t=0}^{\infty} \beta^{t}\left[\alpha\left(y_{t}-\bar{y}\right)^{2}+\left(\pi_{t}-\bar{\pi}\right)^{2}\right]$
} 
advantage of this information and design a better policy. We do not allow for this possibility here, so the CB does not try to actively influence the learning process of agents.

Equations (1)-(6) give rise to a system of the form

$$
x_{t}=A E_{t}^{1} x_{t+1}+B E_{t}^{2} x_{t+1}+C x_{t-1}+D w_{t}+e_{t},
$$

where $x_{t}=\left(y_{t}, \pi_{t}\right)^{\prime}, w_{t}=\left(u_{t}, g_{t}\right)^{\prime}, v_{t}=\left(\tilde{u}_{t}, \tilde{g}_{t}\right)^{\prime}, A$ and $B$ depends on the specific policy implemented by the CB and

$$
\begin{aligned}
C & =\left(\begin{array}{cc}
-\varphi \phi_{1} & 0 \\
-\lambda \varphi \phi_{1} & 0
\end{array}\right) \\
D & =\left(\begin{array}{cc}
-\varphi \phi_{5} & -\varphi \phi_{4}+1 \\
-\lambda \varphi \phi_{5}+1 & -\lambda \varphi \phi_{4}+\lambda
\end{array}\right) .
\end{aligned}
$$

Note that when the optimal policy rule is implemented, the demand shock $g_{t}$ is completely offset by the optimal policy response and the r.h.s. of matrix $D$ becomes zero.

The MSV solution of the model (1)-(6) under homogeneous rational expectations would take the form

$$
x_{t}=P_{1} x_{t-1}+P_{2} w_{t},
$$

where $x_{t-1}$ enters because of policy rule (6). The two groups of agents form expectations according to the two models

$$
\begin{array}{ll}
P L M^{1} & : \quad x_{t}=K_{1} x_{t-1}+K_{2} w_{t} \\
P L M^{2} & : \quad x_{t}=H w_{t},
\end{array}
$$

where we see that while group 1 uses a model that is correctly specified, the other group underparameterizes its model, missing the history dependent component introduced in the economy by the CB. The population of agents is split into the two groups according to the (fixed) proportions $\omega$ and $1-\omega$ : each group independently estimates its own PLM using recursive least squares techniques and then uses it to make forecasts for next period output and inflation.

The specific value taken by the expectational parameter matrices $A$ and $B$ in (8) depends on the policy rule implemented by CB. Three scenario are possible: a) the $\mathrm{CB}$ responds to average expectations; b) the $\mathrm{CB}$ considers only type- 1 expectations; c) the CB considers only type- 2 expectation. The second and third scenario could arise because of the CB unawareness of the existence of different 
expectations or because of its deliberate choice to ignore the expectations of one group of agents. Accordingly, the three policy rules that we consider are:

rule 1:

$$
i_{t}=\phi_{1} y_{t-1}+\phi_{2} E_{t}^{A} \pi_{t+1}+\phi_{3} E_{t}^{A} y_{t+1}+\phi_{4} g_{t}+\phi_{5} u_{t}
$$

rule 2:

$$
i_{t}=\phi_{1} y_{t-1}+\phi_{2} E_{t}^{1} \pi_{t+1}+\phi_{3} E_{t}^{1} y_{t+1}+\phi_{4} g_{t}+\phi_{5} u_{t}
$$

rule 3 :

$$
i_{t}=\phi_{1} y_{t-1}+\phi_{2} E_{t}^{2} \pi_{t+1}+\phi_{3} E_{t}^{2} y_{t+1}+\phi_{4} g_{t}+\phi_{5} u_{t} .
$$

Each of these rules gives rise to different dynamics for the economy. We investigate in particular the issue of determinacy and learnability of the ensuing HEE equilibrium for each case.

\subsection{HEE equilibrium}

\subsubsection{Existence of equilibrium}

In this section we outline the techniques necessary to derive and analyze an HEE, which will then be applied to the economic model introduced above.

The general form of the model to be used is

$$
x_{t}=A E_{t}^{1} x_{t+1}+B E_{t}^{2} x_{t+1}+C x_{t-1}+D w_{t},
$$

where $x_{t}$ is an n-vector of endogenous variables and $w_{t}$ an m-vector of exogenous variables that follow the process

$$
w_{t}=F w_{t-1}+v_{t},
$$

with $F$ a stable $m$ by $m$ matrix and $v_{t}$ a vector of i.i.d. disturbances. $A, B$ and $C$ are $n$ by $n$ matrices, $D$ is a $n$ by $m$ matrix. We have type- 1 agents $\left(\mathrm{PLM}^{1}\right)$ and type-2 agents $\left(\mathrm{PLM}^{2}\right)$ that form expectations $E^{1}$ and $E^{2}$ using the models:

$$
\begin{array}{ll}
\mathrm{PLM}^{1} & : \quad x_{t}=K_{1} x_{t-1}+K_{2} w_{t} \\
\mathrm{PLM}^{2} & : \quad x_{t}=H w_{t},
\end{array}
$$

with $H$ and $K_{2} n$ by $m$ and $K_{1} n$ by $n$ matrices. The ensuing expectations for the endogenous variables are

$$
\begin{aligned}
& E_{t}^{1} x_{t+1}=K_{1}^{2} x_{t-1}+\left(K_{1} K_{2}+K_{2} F\right) w_{t} \\
& E_{t}^{2} x_{t+1}=H F w_{t},
\end{aligned}
$$


that substituted in (15) give the reduced form

$$
x_{t}=\left(C+A K_{1}^{2}\right) x_{t-1}+\left(B H F+A K_{1} K_{2}+A K_{2} F+D\right) w_{t} .
$$

Using the techniques developed by Evans and Honkapohja (2001), we can build a direct map from PLM ${ }^{1}$ to the ALM and derive ordinary differential equations that mimic the limiting asymptotic behavior for the RLS algorithms.

The maps from PLM $^{1}$ to ALM are

$$
\begin{aligned}
& K_{1} \rightarrow C+A K_{1}^{2} \\
& K_{2} \rightarrow B H F+A K_{1} K_{2}+A K_{2} F+D,
\end{aligned}
$$

and the ensuing differential equations are

$$
\begin{aligned}
\dot{K}_{1} & =A K_{1}^{2}-K_{1}+C \\
\dot{K}_{2} & =B H F+A K_{1} K_{2}+A K_{2} F+D-K_{2} .
\end{aligned}
$$

Fixed points of these differential equations are equilibrium values for $K_{1}$ and $K_{2}$. Equation (24) is a matrix quadratic differential equation, and no general method is available for obtaining the general set of solutions, that are in number $\left(\begin{array}{c}2 n \\ n\end{array}\right)$ for an $n$ by $n K_{1}$ matrix (see Evans and Honkapohja (2001), p. 230). We thus follow McCallum (1983) and look for the MSV solution, which is unique by construction.

Since our matrix $A$ is not invertible, we can not use the procedure developed in McCallum (1983), but need instead to use the more general techniques outlined in McCallum (1998) and Uhlig (1999). We will follow closely Uhlig (1999) in our derivation of a solution for $K_{1}$. The matrix quadratic equation to be solved is

$$
A K_{1}^{2}-K_{1}+C=0,
$$

which can be rewritten as a system

$$
\left(\begin{array}{cc}
A & 0_{n} \\
0_{n} & I_{n}
\end{array}\right)\left(\begin{array}{l}
K_{1}^{2} \\
K_{1}
\end{array}\right)=\left(\begin{array}{cc}
I_{n} & -C \\
I_{n} & 0_{n}
\end{array}\right)\left(\begin{array}{c}
K_{1} \\
I
\end{array}\right)
$$

Defining the two matrices

$$
\Xi=\left(\begin{array}{cc}
I_{n} & -C \\
I_{n} & 0_{n}
\end{array}\right), \Delta=\left(\begin{array}{cc}
A & 0_{n} \\
0_{n} & I_{n}
\end{array}\right)
$$

we need to find the eigenvalues of $\Xi$ with respect to $\Delta$ and then select those eigenvalues that together with the corresponding eigenvectors are to be used in 
the solution. As we are looking for the MSV solution, we have to choose the eigenvalues that approach zero as $C \rightarrow 0$. Keeping track of them as we let a constant that pre-multiplies $C$ go from 1 to 0 we are able to identify the relevant eigenvalues. Let $\Lambda$ be the diagonal matrix with these $n$ eigenvalues on the main diagonal, and $\Omega$ the matrix of the corresponding eigenvectors, we have

$$
K_{1}=\Omega \Lambda \Omega^{-1},
$$

the MSV solution for $K_{1}$.

Going back to the matrix differential equation (25), it is clear that this can not be analyzed independently from $H$. We therefore need to consider simultaneously also the learning process for the second group of agents. In this case, though, it is not possible to build a direct map from the PLM ${ }^{2}$ to the ALM and we need to project the ALM onto the space of the $\mathrm{PLM}^{2}$. The stochastic recursive algorithm (SRA) representing RLS learning for this case is

$$
\begin{aligned}
H_{t}^{\prime} & =H_{t-1}^{\prime}+t^{-1} R_{t}^{-1} w_{t-1}\left(x_{t-1}-\hat{x}_{t-1}\right)^{\prime} \\
R_{t} & =R_{t-1}+t^{-1}\left(w_{t-1} w_{t-1}-R_{t-1}\right)
\end{aligned}
$$

where $x_{t-1}$ is given by (21) moved backwards one period and

$$
\hat{x}_{t-1}=H_{t-1} w_{t-1} .
$$

$R_{t}$ is an $m$ by $m$ matrix, and we make the transformation $R_{t}=S_{t-1}$, as on the r.h.s. of the SRA we must have only variables dated $t-1$ or earlier. The associated ODE that governs stability of this SRA is

$$
\frac{d \Phi}{d \tau}=h(\Phi)=\lim _{t \rightarrow \infty} E Q\left(t, \Phi, z_{t}\right)
$$

where $\Phi=\operatorname{vec}\left(H^{\prime} S\right), z_{t}=\left(w_{t-1}^{\prime}, x_{t-2}^{\prime}\right)^{\prime}$ and the expectation is taken over the invariant distribution of $z_{t}$ for fixed $\Phi$. It is easier to work separately with

$$
\begin{aligned}
h_{H}(\Phi) & =\lim _{t \rightarrow \infty} E Q_{H}\left(t, \Phi, z_{t}\right) \\
h_{S}(\Phi) & =\lim _{t \rightarrow \infty} E Q_{S}\left(t, \Phi, z_{t}\right)
\end{aligned}
$$

where

$$
\begin{aligned}
Q_{H}\left(t, \Phi, z_{t}\right) & =\left(S^{-1} w_{t-1}\left(x_{t-1}-\hat{x}_{t-1}\right)^{\prime}\right) \\
Q_{S}\left(t, \Phi, z_{t}\right) & =\left(\frac{t}{t+1}\right)\left(w_{t-1} w_{t-1}^{\prime}-S\right) .
\end{aligned}
$$


By substituting in $x_{t-1}$ from (21) and $\hat{x}_{t-1}$ from (32) we get:

$$
\begin{array}{r}
Q_{H}\left(t, \Phi, z_{t}\right)=S^{-1} w_{t-1}\left(\left(A K_{1}^{2}+C\right) x_{t-2}+\left(B H F+A K_{1} K_{2}+A K_{2} F+\right.\right. \\
\left.D-H) w_{t-1}\right)^{\prime} .
\end{array}
$$

It thus follows

$$
\begin{array}{r}
h_{H}(\Phi)=\lim _{t \rightarrow \infty} E S^{-1} w_{t-1}\left(\left(A K_{1}^{2}+C\right) x_{t-2}+\left(B H F+A K_{1} K_{2}+A K_{2} F+\right.\right. \\
\left.D-H) w_{t-1}\right)^{\prime}
\end{array}
$$

and

$$
h_{S}(\Phi)=\lim _{t \rightarrow \infty}\left(\frac{t}{t+1}\right) E\left(w_{t-1} w_{t-1}-S\right)=M-S,
$$

where $M=\lim _{t \rightarrow \infty} E w_{t-1} w_{t-1}=\lim _{t \rightarrow \infty} E w_{t} w_{t}^{\prime}=\Sigma_{w}$ is he asymptotic variance covariance matrix for the exogenous variables. From (40) we can see that $S \rightarrow M$, which leads to

$$
h_{H}(\Phi)=\left(B H F+A K_{1} K_{2}+A K_{2} F+D-H\right)^{\prime}+\left(\Sigma_{w}\right)^{-1} \Sigma_{w x}\left(C+A K_{1}^{2}\right)^{\prime},
$$

where $\Sigma_{w x}=\lim _{t \rightarrow \infty} E w_{t-1} x_{t-2}^{\prime}$ is determined endogenously and depends on the parameters in the PLMs:

$$
\begin{array}{r}
E w_{t-1} x_{t-2}^{\prime}=E\left(F w_{t-2}+v_{t-1}\right)\left(\left(C+A K_{1}^{2}\right) x_{t-3}+\left(B H F+A K_{1} K_{2}+\right.\right. \\
\left.\left.A K_{2} F+D\right) w_{t-2}\right)^{\prime} .
\end{array}
$$

Given that the variables are taken to be (asymptotically) stationary, we have that

$$
\lim _{t \rightarrow \infty} E\left(w_{t-1} x_{t-2}^{\prime}\right)=\lim _{t \rightarrow \infty} E\left(w_{t-2} x_{t-3}^{\prime}\right)
$$

and thus

$$
\Sigma_{w x}=F \Sigma_{w x}\left(C+A K_{1}^{2}\right)^{\prime}+F \Sigma_{w}\left(B H F+A K_{1} K_{2}+A K_{2} F+D\right)^{\prime} I,
$$

which after being vectorized can be written as

$$
\operatorname{vec}\left(\Sigma_{w x}\right)=\left[I-\left(C+A K_{1}^{2}\right) \otimes F\right]^{-1}\left[I \otimes F \Sigma_{w}\right] \operatorname{vec}\left(B H F+A K_{1} K_{2}+A K_{2} F+D\right)^{\prime} .
$$

Vectorizing (41) we get

$$
\begin{aligned}
\operatorname{vec}\left[h_{H}(\Phi)\right]=\operatorname{vec}\left(B H F+A K_{1} K_{2}+A K_{2} F+D-H\right)^{\prime}+ \\
{\left[\left(C+A K_{1}^{2}\right) \otimes\left(\Sigma_{w}\right)^{-1}\right] \operatorname{vec}\left(\Sigma_{w x}\right), }
\end{aligned}
$$


and substituting $\operatorname{vec}\left(\Sigma_{w x}\right)$ into (46) we obtain

$$
\begin{array}{r}
\operatorname{vec}\left[h_{H}(\Phi)\right]=\left(\left(C+A K_{1}^{2}\right) \otimes\left(\Sigma_{w}\right)^{-1}\right)\left(I-\left(C+A K_{1}^{2}\right) \otimes F\right)^{-1}\left(I \otimes F \Sigma_{w}\right) \\
+I) \operatorname{vec}\left(B H F+A K_{1} K_{2}+A K_{2} F+D\right)^{\prime}-v e c H^{\prime}
\end{array}
$$

which can be rewritten as

$$
\begin{aligned}
& \operatorname{vec}\left[h_{H}(\Phi)\right]=\left[\left(C+A K_{1}^{2}\right) \otimes\left(\Sigma_{w}\right)^{-1}\right)\left(I-\left(C+A K_{1}^{2}\right) \otimes F\right)^{-1}\left(I \otimes F \Sigma_{w}\right) \\
& \quad+I]\left[\left(B \otimes F^{\prime}\right) v e c H^{\prime}+\left(\left(A K_{1} \otimes I\right)+\left(A \otimes F^{\prime}\right)\right) v e c K_{2}^{\prime}+v e c D^{\prime}\right]-v e c H^{\prime}
\end{aligned}
$$

or more concisely

$$
\begin{array}{r}
v e c\left[h_{H}(\Phi)\right]=(I+P)\left[\left(B \otimes F^{\prime}\right) v e c H^{\prime}+\left(\left(A K_{1} \otimes I\right)+\left(A \otimes F^{\prime}\right)\right) v e c K_{2}^{\prime}+\operatorname{vec} D^{\prime}\right] \\
-v e c H^{\prime}, \quad(49)
\end{array}
$$

where

$$
P=\left(\left(C+A K_{1}^{2}\right) \otimes\left(\Sigma_{w}\right)^{-1}\right)\left(I-\left(C+A K_{1}^{2}\right) \otimes F\right)^{-1}\left(I \otimes F \Sigma_{w}\right) .
$$

We are looking for an equilibrium point $\left(\bar{K}_{1}, \bar{K}_{2}, \bar{H}\right)$ of the system formed by (24), (25) and (49). Equation (24) has already been solved above: given a solution for $K_{1},(25)$ and (49) give unique solutions for $K_{2}$ and $H$. These two equations form the system

$$
\left(\begin{array}{l}
v e c \dot{K}_{2}^{\prime} \\
v e c \dot{H}^{\prime}
\end{array}\right)=\Gamma\left(\begin{array}{l}
v e c K_{2}^{\prime} \\
v e c H^{\prime}
\end{array}\right)+\Psi
$$

where

$$
\begin{aligned}
\Gamma & =\left(\begin{array}{cc}
\left(A \bar{K}_{1} \otimes I\right)+\left(A \otimes F^{\prime}\right)-I & \left(B \otimes F^{\prime}\right) \\
\left(A \bar{K}_{1} \otimes I\right)+\left(A \otimes F^{\prime}\right) & (I+P)\left(B \otimes F^{\prime}\right)-I
\end{array}\right) \\
\Psi & =\left(\begin{array}{c}
\text { vec } D^{\prime} \\
(I+P) \text { vec } D^{\prime}
\end{array}\right) .
\end{aligned}
$$

Imposing it equal to zero we get the solution

$$
\left(\begin{array}{l}
\operatorname{vec} K_{2}^{\prime} \\
\operatorname{vec} H^{\prime}
\end{array}\right)=-\Gamma^{-1} \Psi
$$

from which we can reconstruct matrices $K_{2}$ and $H$. 


\subsubsection{E-stability}

The concept we use to assess the learnability of the equilibrium is the E-stability property proposed by Evans and Honkapohja (2001). An E-stable equilibrium is one that can be learned, under some general conditions, with recursive learning schemes such as recursive least squares.

To investigate E-stability for the HEE we need to focus our attention on the differential equations that represent the maps from the PLMs to the ALM for the three matrices of parameters $K_{1}, K_{2}$ and $H$, i.e., to equations (24), (25) and (49). Equation (24) is a matrix quadratic differential equation independent of $K_{2}$ and $H$, so it can be analyzed autonomously: convergence of the learning process for $K_{1}$ requires the real part of the eigenvalues of the derivative of $(24)$ w.r.t. $K_{1}$ to be negative. The relevant Jacobian is (see Evans and Honkapohja (2001), p. 231)

$$
\bar{K}_{1}^{\prime} \otimes A+I \otimes\left(A \bar{K}_{1}-I\right) .
$$

Convergence of the learning dynamics for $K_{2}$ and $H$ must be analyzed together, using the system (51): E-stability requires that the eigenvalues of matrix $\Gamma$ have all negative real part.

Proposition 1. The HEE is E-stable iff the matrices $\bar{K}_{1}^{\prime} \otimes A+I \otimes\left(A \bar{K}_{1}-I\right)$ and $\Gamma$ have all eigenvalues with negative real part.

\subsubsection{Determinacy of equilibrium}

As our model, in equilibrium, has both rational and non-rational expectations, the standard procedure usually employed to check for determinacy can not be directly applied to the HEE. Determinacy must be intended here in a restricted sense: the HEE is said to be unique if it is the only stable equilibrium in which type- 1 agents are rational and type- 2 agents form their expectations by using the underparameterized model (18) with learning scheme (30) and (31) at its resting point. Our procedure has nothing to say about the possibility of having other stable HEE in which type-2 agents use a different form of underparameterized PLM or a different learning scheme. With this caveat, we can follow the procedure suggested by Branch and McGough (2005) and associate a RE model to the model with heterogenous expectations developed here. This allows us to carry out the analysis of determinacy for the HEE in the associated RE model through the standard techniques developed by Blanchard and Kahn (1980). An HEE is said to be determinate iff the associated RE model is determinate. 
In system (15) there are two different expectational operators: while $E_{t}^{1}$ represents expectations that, in equilibrium, turn out to be rational $(E), E_{t}^{2}$ indicates expectations that are not fully rational in the HEE, though they represent a fixed point for the associated learning scheme. We thus need to substitute out $E_{t}^{2} x_{t+1}$ from (15) and replace it with the corresponding equation (20):

$$
x_{t}=A E_{t} x_{t+1}+(B H F+D) w_{t}+C x_{t-1}+e_{t} .
$$

Rewriting (56) as a first order system we obtain

$$
\left(\begin{array}{cc}
I_{n} & 0_{n} \\
I_{n} & -C
\end{array}\right)\left(\begin{array}{c}
x_{t} \\
x_{t-1}
\end{array}\right)=\left(\begin{array}{cc}
0_{n} & I_{n} \\
A & 0_{n}
\end{array}\right) E_{t}\left(\begin{array}{c}
x_{t+1} \\
x_{t}
\end{array}\right)+\text { other }
$$

which can now be analyzed by applying the standard techniques developed by Blanchard and Kahn (1980). The relevant matrix for determinacy is

$$
\Upsilon=\left(\begin{array}{cc}
I_{n} & 0_{n} \\
I_{n} & -C
\end{array}\right)^{-1}\left(\begin{array}{cc}
0_{n} & I_{n} \\
A & 0_{n}
\end{array}\right) .
$$

The HEE is determinate iff the number of eigenvalues of $\Upsilon$ that lie inside the unit circle is equal to the number of non-predetermined variables in the system, i.e., to $n$.

\subsection{Equilibria under different policy specifications}

We can now apply the above techniques to the model presented in Section 3.1. For each of the three policy rules (12), (13), (14) we compute the numerical values for the matrices $\mathrm{K}_{1} \mathrm{~K}_{2}$ and $\mathrm{H}$ in equilibrium, and check whether determinacy and E-stability of each HEE obtain.

Then, for those equilibria that we find to be E-stable, we evaluate the tradeoff in terms of inflation and output variability. This is done by computing the efficient policy frontier (see Woodford 2003, ch. 6), which shows the implied tradeoff between asymptotic variance of inflation and output for different values of the policy parameter $\alpha$.

The aim of this analysis is to determine whether the action of the CB can affect the equilibrium outcome for the economy, and in that case, which of the possible outcomes is preferred by the policymaker. If the $\mathrm{CB}$, by implementing a specific policy, can move the economy from one equilibrium to another (through the learning of agents), then it should try to drive the economy towards the equilibrium that presents the most favorable trade-off in terms of inflation and output variability. 


\subsubsection{Policy 1}

When CB implements (12), the system given by (1), (2) and (12) can be mapped into (15) using (3), with

$$
\begin{aligned}
& A=\omega \bar{A} \\
& B=(1-\omega) \bar{A},
\end{aligned}
$$

where

$$
\bar{A}=\left(\begin{array}{cc}
1-\varphi \phi_{3} & \varphi\left(1-\phi_{2}\right) \\
\lambda\left(1-\varphi \phi_{3}\right) & \lambda \varphi\left(1-\phi_{2}\right)+\beta
\end{array}\right) .
$$

As for matrices $C$ and $D$, these are the same for all the three cases considered, and remain as described by (9). Given these matrices, and using the techniques described above, we can find the numerical values for the equilibrium values $H$, $K_{1}, K_{2}$, for each of the three parameterizations proposed above.

With these values, we then study the properties of the equilibrium: numerical results show that the HEE is both unique and E-stable for each of the three parameterizations considered. We set $\omega=.5$ and $\alpha=.1$ as baseline case, but we then check how things change when different proportions of agents in the economy are allowed, going from a situation in which the large majority of agents are of type-1 $(\omega=.9)$ to one in which the population is composed largely of type- 2 agents $(\omega=.1)$. Numerical results show that in all the cases the HEE is E-stable and unique, for all the three parameterizations used.

\subsubsection{Policy 2}

When CB implements (13), the system given by (1), (2) and (13) can be mapped into (15), with:

$$
\begin{aligned}
& A=\left(\begin{array}{cc}
\omega-\varphi \phi_{3} & \varphi\left(\omega-\phi_{2}\right) \\
\lambda\left(\omega-\varphi \phi_{3}\right) & \lambda \varphi\left(\omega-\phi_{2}\right)+\beta \omega
\end{array}\right) \\
& B=\left(\begin{array}{cc}
1-\omega & \varphi(1-\omega) \\
\lambda(1-\omega) & \lambda \varphi(1-\omega)+\beta(1-\omega)
\end{array}\right) .
\end{aligned}
$$

With these new matrices, we compute the equilibrium values for $H, K_{1}, K_{2}$ : numerical results show that the HEE is both unique and E-stable for all the three parameterizations proposed. Again, when we experiment with $\omega=.1$ and .9 , we find that results do not change. 


\subsubsection{Policy 3}

When CB implements (14), the system given by (1), (2) and (14) can be mapped into (15), with:

$$
\begin{aligned}
A & =\left(\begin{array}{cc}
\omega & \varphi \omega \\
\lambda \omega & \lambda \varphi \omega+\beta \omega
\end{array}\right) \\
B & =\left(\begin{array}{cc}
(1-\omega)-\varphi \phi_{3} & \varphi(1-\omega)-\varphi \phi_{2} \\
\lambda\left(1-\omega-\varphi \phi_{3}\right) & \lambda\left[\varphi(1-\omega)-\varphi \phi_{2}\right]+\beta(1-\omega)
\end{array}\right) .
\end{aligned}
$$

We again find the equilibrium values for the parameters in the ALM. Numerical results show that the HEE is now not unique, and moreover is E-unstable for all the parameterizations proposed when $\omega$ is equal to .5 . With $\omega=.9$ results do not change, but using $\omega=.1$ the HEE becomes unique and E-stable for the CGG and MN parameterizations. This result needs further attention, as it says that when CB responds to type-2 expectations agents are not able to learn the relevant equilibrium unless there is only a small number of type- 1 agents in the economy, in which case the equilibrium might become learnable (and unique), depending on the parameterization used.

\subsection{Equilibria comparison}

Our investigations above showed that both policy 1 and policy 2 lead to an HEE that is unique and E-stable. Results for policy 3 are ambiguous, but in the vast majority of the cases considered the resulting HEE is indeterminate and not Estable. We therefore restrict our welfare analysis to the equilibria that emerge under policy 1 and policy 2 .

For this purpose, we compute the efficient policy frontier for each of the two HEE. The frontier shows the trade off in terms of output and inflation variability for different values of the weight parameter $\alpha$ in the loss function for the central bank. From Figure 1 we can see that the CB faces a more favorable trade off when implementing policy 2 (i.e., when responding only to type- 1 expectations), as the policy frontier in this case lies always below the frontier obtained with policy 1 . This is especially true for high values of $\alpha$, when the gap between the two frontiers is larger.

In order to understand this result, we compute the impulse-response functions for various variables after a one-unit cost push shock to the AS equation. Figure 2 and 3 show the impulse response for output and inflation: we can see that under 
policy 1 inflation grows less but output drops more than under policy 2 (here, and in all the following impulse-response calculations, $\alpha$ is set at .5. Results don't change for different values of $\alpha$ ). This result explains why the higher is $\alpha$ (i.e., the more the $\mathrm{CB}$ cares about output variability), the larger is the gain from using policy 2 .

If we then look at the policy response, we can see that the interest rate raises more under policy 2 (Figure 4), but the policy stance (real interest rate, i.e., $i-E_{t}^{A} \pi_{t+1}$ ) is actually more restrictive under policy 1 (Figure 5). This happens because average expectations for inflation (Figure 6) under policy 1 are lower than under policy 2, and thus the real interest rate ends up being higher under policy 1 , even with a lower nominal interest rate.

Another important point to note is that under policy 2 inflation expectations of each type rise more and those for output drop less than the same type under policy 1 (Figure 8 and 9). Moreover, type-1 expectations for inflation under policy 2 , those upon which this policy is based, rise more than average expectations for inflation under policy-1, thosethat enter in this policy, while type-1 output expectations under policy 2 drop less than average expectations under policy 1: as a result, the nominal interest rate raises more under policy 2 .

It is important to note that there is a circular dependency: not only expectations affect policy, but also policy affects expectations. To summarize, simulations show that: a) under policy 1, output drops more but inflation rises less than under policy 2 ; b) under policy 1 , interest rate raises less, but policy is more restrictive because average expectations for inflation remain lower; as a consequence, inflation raises less and output drops more; in other words, with lower expectations for inflation policy 1 is less responsive to supply shocks, but then the stance is more restrictive because lower expectations for inflation mean a higher real interest rate; c) consistently, with policy 1 inflation expectations of both types rise less, and those for output drop more; d) type-2 expectations for inflation rise more, and those for output drop less, than type-1 do, under both policies; we would therefore expect interest rates to increase more under policy 1, but e) with policy 2, expectations (of both types) call for a stronger reaction: that's why interest rates increase more in this case; f) type- 2 expectations call for a stronger policy response, under both policies.

A key point here is that type-2 expectations call for a stronger policy than type- 1 expectations do, because inflation is expected to raise more and output to drop less. At the same time, with respect to policy 2, policy 1 makes expectations for inflation rise less, and those for output drop more, for both groups of agents, 
which in turn call for a milder policy. What we observe in equilibrium is a sort of fixed point between expectations and policy reaction: expectations affect the stance of policy, and policy affects the magnitude of expectations. It is not possible to disentangle the two causal relations.

What the policy frontier allows us to see is that the equilibrium dynamics resulting from the interactions between expectations and policy reaction generate a combination of output and inflation variability that is more favorable under policy 2 , when the CB neglects the inaccurate expectations. Those expectations, in fact, though they do affect the economy, do not give a precise picture of the future state of the economy, and thus call for a policy that would generate unnecessary volatility in the economy.

\section{Conclusions}

Recent studies in monetary policy have suggested that the policymaker can better stabilize the economy by taking into account, when setting the interest rate, also the expectations held by the private sector.

In this paper, building on this literature, we have extended the analysis to a situation in which private agents hold heterogeneous expectations: while some of them recognize the role played by the CB in the economy, and thus are able to select the correct variables to include in the model they use to form expectations, the rest of the agents overlook at the history dependence introduced into the economy by the commitment policy of the $\mathrm{CB}$ and therefore use a misspecified model. Given the forward-looking nature of the economy, those expectations then feed back into the system and determine its future state.

The main point that emerges is that heterogeneity in expectations matter, and that simply assuming it away by taking averages might be misleading. In using private sector's expectations to decide its policy action, the CB should keep this in mind and carefully consider which expectations to use. On this point, our results seem to show that the CB should consider only the "good" expectations, and disregard the "bad" ones.

An implication of this result is that if the CB has accurate (rational) expectations, it can just use its own forecasts in order to set the policy instrument and forget about the private sector and its heterogeneity altogether.

Given the heterogeneity of expectations we can observe in the real world, and the importance that expectations have in shaping the economy, we think this is a topic that deserves great consideration, and we hope that this paper will stimulate 
others to investigate this and related issues.

\section{Figures}

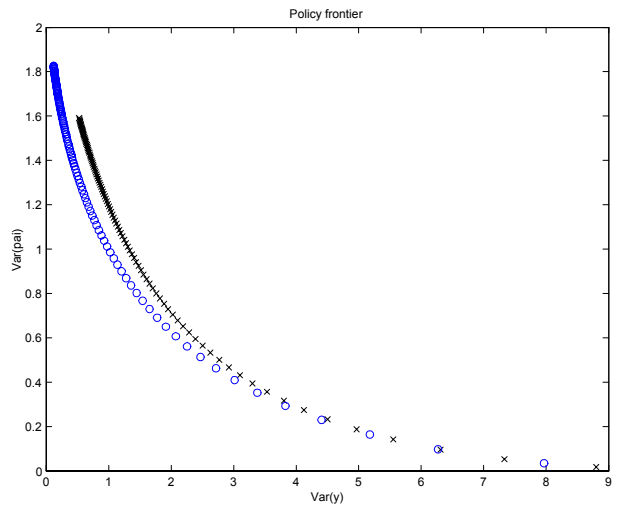

Figure 1: Policy frontiers. Black line (crosses) with policy 1, blue line (circles) with poilicy 2 .

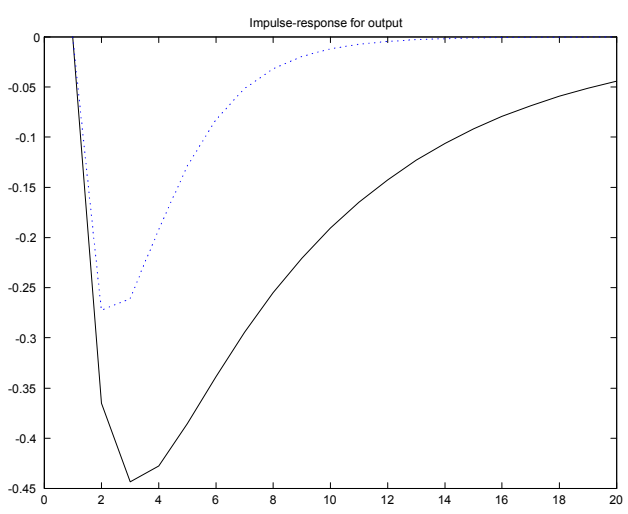

Figure 2: Inpulse-response for output under policy 1 (continuous line) and 2 (dotted line). 


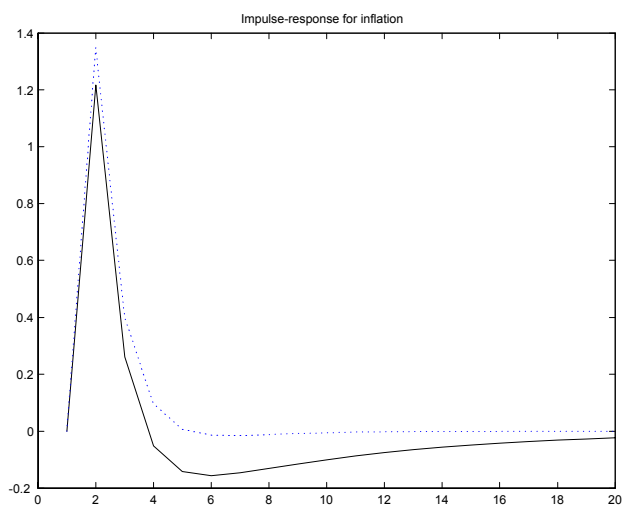

Figure 3: Inpulse-response for inflation under policy 1 and 2.

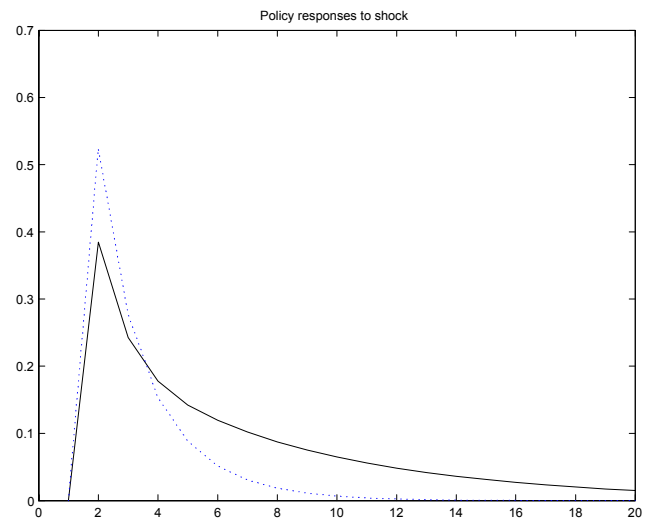

Figure 4: Policy response under policy 1 and 2.

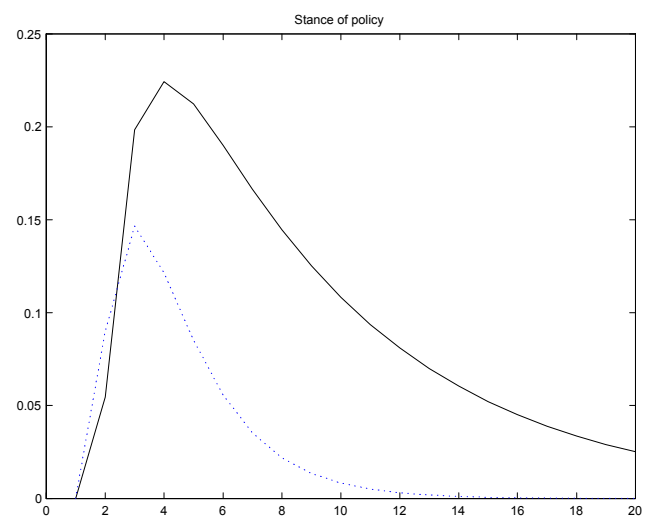

Figure 5: Stance of policy, under policy 1 and 2. 


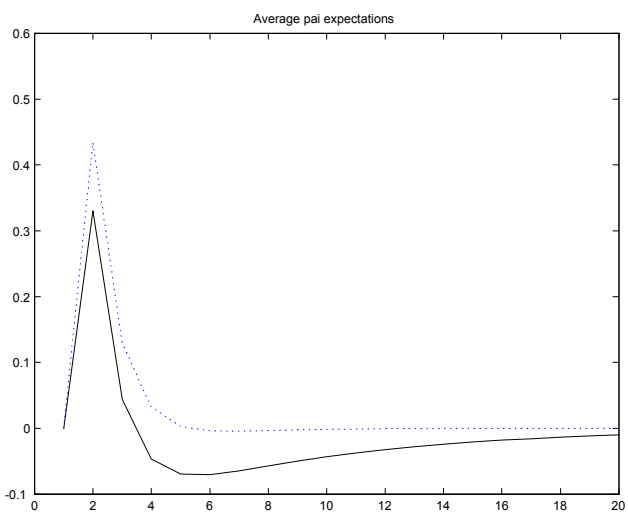

Figure 6: Average expectations for inflation, under policy 1 and 2.

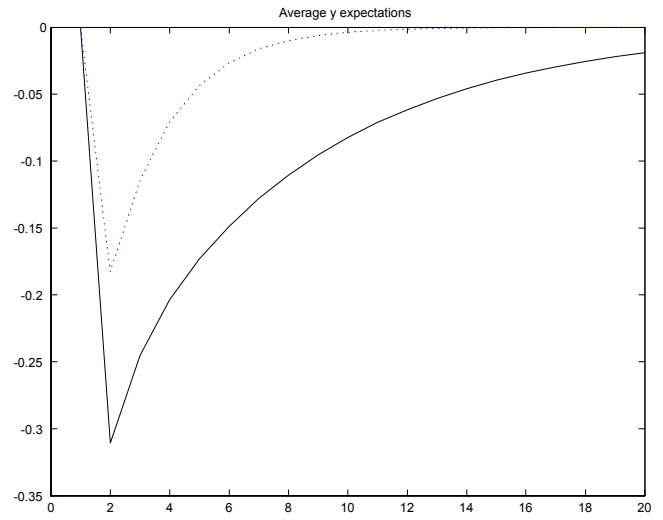

Figure 7: Average expectations for output, under policy 1 and 2. 


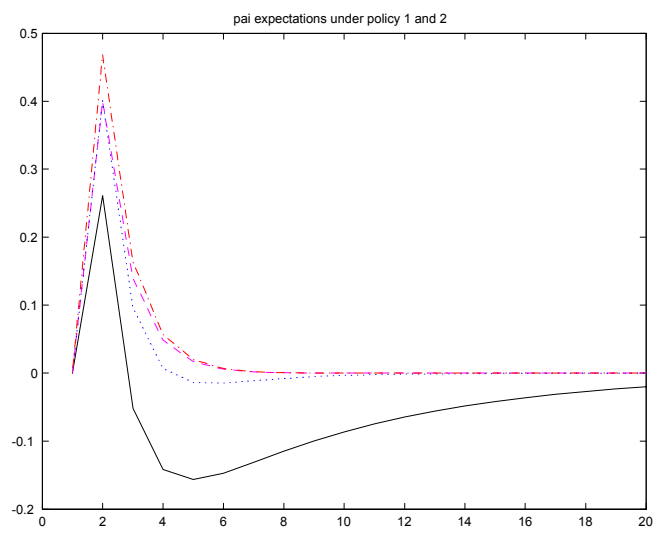

Figure 8: Inflation expectations. Black continuous line: type-1, under policy1; blue dotted line: type-1, under policy 2; magenta dashed line: type-2, under policy 1; red dashed dotted line: type-2, under policy 2 .

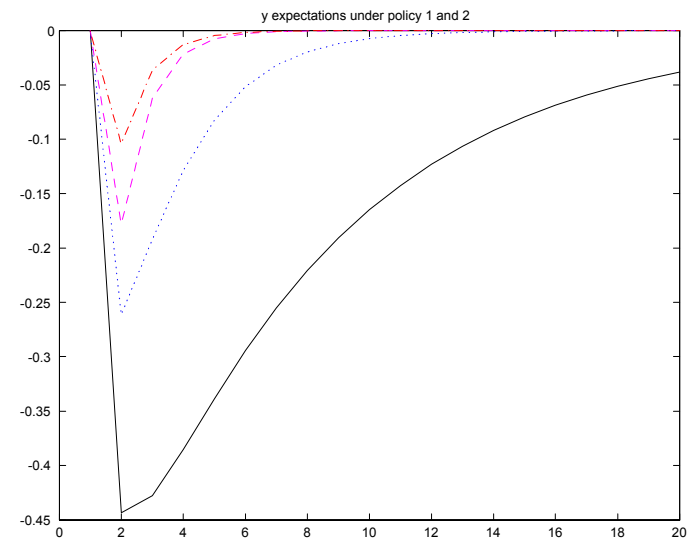

Figure 9: Output expectations. Black continuous line: type-1, under policy1; blue dotted line: type-1, under policy 2; magenta dashed line: type-2, under policy 1; red dashed dotted line: type-2, under policy 2. 


\section{References}

[1] Berardi, Michele (2007) "Heterogeneity and misspecifications in learning", Journal of Economic Dynamics and Control, forthcoming.

[2] Berardi, Michele and John Duffy (2007), "The Value of Transparency when Agents are Learning", European Journal of Political Economy, forthcoming.

[3] Blanchard, Olivier J. and Charles M. Kahn (1980), "The Solution of Linear Difference Models under Rational Expectations", Econometrica, 48, 5, 13051311.

[4] Branch, William A. and Bruce McGough (2005), "Multiple Equilibria in Heterogeneous Expectations Models", forthcoming, Contributions in Macroeconomics.

[5] Clarida, Richard, Jordi Gali and Mark Gertler (1999), "The Science of Monetary Policy: A New Keynesian Perspective", Journal of Economic Literature, 37, 1661-1707.

[6] Clarida, Richard, Jordi Gali and Mark Gertler (2000), "Monetary Policy Rules and Macroeconomic Stability: Evidence and Some Theory", Quarterly Journal of Economics, 115, 147-180.

[7] Evans, George W. and Seppo Honkapohja (2001), Learning and Expectations in Macroeconomics. Princeton University Press, Princeton, New Jersey.

[8] Evans, George W. and Seppo Honkapohja (2003a), "Expectations and the Stability Problem for Optimal Monetary Policies", Review of Economic Studies, 70, 807-824.

[9] Evans, George W. and Seppo Honkapohja (2003b), "Monetary Policy, Expectations and Commitment", working paper.

[10] Ferrero, Giuseppe (2004), "Monetary Policy and the Transition to Rational Expectations", working paper.

[11] Honkapohja, Seppo and Kaushik Mitra (2006), "Learning Stability in Economics with Heterogeneous Agents", Review of Economic Dynamics, 9, 284309. 
[12] McCallum, Bennett T. (1983), "On Non-Uniqueness in Rational Expectations Models: An Attempt at Perspective", Journal of Monetary Economics, 11, 139-168.

[13] McCallum, Bennett T. (1998), "Solutions to Linear Rational Expectations Models: A Compact Exposition", Economics Letters, 61, 143-147.

[14] McCallum, Bennett T. and Edward Nelson (1999), "Performance of Operational Policy Rules in an Estimated Semi-Classical Model", in John B. Taylor (ed.) (1999), Monetary Policy Rules, University of Chicago Press, Chicago.

[15] Molnar, Krisztina and Sergio Santoro (2005), "Optimal Monetary Policy When Agents Are Learning", working paper.

[16] Uhlig, Harald (1999), "A Toolkit for Analyzing Nonlinear Dynamic Stochastic Models Easily", Computational Methods for the Study of Dynamic Economies, edited by R. Marimon and A. Scott, Oxford University Press.

[17] Woodford, Michael (1999), "Optimal Monetary Policy Inertia", NBER Working paper 7261, July.

[18] Woodford, Michael (2000), "Pitfalls of Forward-Looking Monetary Policy," American Economic Review, 90(2), 100-104.

[19] Woodford, Michael (2003), Interest and Prices. Foundations of a Theory of Monetary Policy, Princeton University Press, Princeton, NJ. 